\title{
Factores familiares asociados al inicio sexual temprano en adolescentes consultantes en un centro de salud sexual y reproductiva en Santiago de Chile
}

\author{
ELECTRA GONZÁLEZ A. ${ }^{a}$, TEMÍSTOCLES MOLINA G. \\ ADELA MONTERO V., VANIA MARTÍNEZ N.
}

\section{Factors associated with early sexual activity among Chilean adolescents}

\begin{abstract}
Background: Nowadays, adolescent population begins sexual activity earlier, a behavior with negative psychological and social consequences. Aim: To determine the association between family factors and early sexual activity in adolescents of low and middle socioeconomic level. Material and Methods: A sample of 3,210 adolescents, who confidentially requested care in a sexual and reproductive health university center, between 2000 and 2007, was analyzed. Adolescents who started sexual activity before 15 years of age, and those who started sexual activity after 15 years of age, were compared. Data was collected from structured interviews conducted at the adolescent's first visit. Logistic regression was used to identify family factors associated with early sexual initiating in both, men and women. Results: Family factors associated with early sexual activity start were not being raised by both parents, a poor parent-child relationship, a poor family communication, mothers with a history of adolescent motherhood, mothers employed outside the home and family dysfunction. Among women, the variables associated with a higher risk of early sexual debut were not married parents and history of adolescent fatherhood record among parents. Among men, these variables were permissions without restriction during the week and punishment when family rules were broken. Conclusions: Family factors must be considered in the design of strategies to prevent early sexual activity.
\end{abstract}

(Rev Med Chile 2013; 141: 313-319).

Key words: Adolescent; Family relations; Sexual behavior; Sexuality.
${ }^{1}$ Centro de Medicina Reproductiva y Desarrollo Integral de la Adolescencia. Facultad de Medicina. Universidad de Chile. ${ }^{a}$ Asistente Social, Master in Population Research. ${ }^{\mathrm{b}}$ Bioestadístico, Magíster en Bioestadísticas.

Recibido el 18 de enero de 2012, aceptado el 26 de octubre de 2012.

Correspondencia a: Electra González A, Centro de Medicina Reproductiva y Desarrollo Integral de la Adolescencia (CEMERA), Facultad de Medicina, Universidad de Chile Prof. Alberto Zañartu 1030, Independencia. Fax 56-2-7356512. E-mail: evgonzalez@med. uchile.cl,cemera@med.

uchile.cl
E n Chile, como en muchos países, los adolescentes están iniciando actividad sexual a edades más tempranas que en el pasado y en su mayoría sin usar métodos anticonceptivos seguros. La edad promedio de inicio de la actividad sexual varía entre 15,7 y 16,3 años para los hombres y entre 15,5 y 17,1 años para las mujeres, observándose principalmente que los hombres y mujeres de menor nivel socioeconómico tienden a iniciarse sexualmente a edades más tempranas ${ }^{1,2}$. Esta actividad sexual temprana y no protegida expone a los adolescentes a infecciones de trans- misión sexual y a embarazos no planeados a más temprana edad. En nuestro país, el 15\% del total de nacidos vivos corresponde a madres menores de 20 años, solteras y en condiciones económicas deficitarias. Este problema es particularmente preocupante en las menores de 15 años $^{3-4}$. Entre las consecuencias negativas de la maternidad adolescente, se incluye pobres condiciones de salud para la madre e hijo y una mayor dependencia de la familia de origen y de programas sociales ${ }^{3-5}$. En nuestro país la actividad sexual adolescente no es reconocida ni aceptada, lo cual puede hacer más 
difícil cualquier esfuerzo para proveer servicios en salud sexual adecuados a sus reales necesidades ${ }^{3-6}$. Reducir el embarazo adolescente no deseado y las infecciones de transmisión sexual, incluido el VIH/SIDA, son metas de políticas públicas en Chile desde hace décadas, sin embargo, hasta ahora las estrategias utilizadas han sido poco exitosas ${ }^{4}$.

Si bien la actividad sexual adolescente es un fenómeno multifactorial, los factores familiares juegan un rol primordial ${ }^{7-11}$, y deberían ser considerados también en el diseño de políticas públicas dirigidas a postergar el inicio sexual en los adolescente. De acuerdo a lo reportado en la literatura internacional, la crianza por ambos padres y la religiosidad parental se asocian significativamente a la postergación del inicio sexual en hombres y mujeres ${ }^{12-15}$. Los adolescentes que viven en familias más numerosas tienen más riesgo de inicio sexual temprano, ya que los padres pueden disponer de menos tiempo para monitorear el comportamiento de sus hijos ${ }^{15}$. Sin embargo, otros estudios muestran que, el mayor número de hijos puede enfatizar la disciplina y la manutención de orientaciones estrictas por parte de los padres ${ }^{14-16}$. Por otro lado, los padres que sostienen actitudes restrictivas hacia la sexualidad adolescente organizan sus propias actividades de tal manera que les permita supervisar y controlar mejor el comportamiento de sus hijos, resultando en una menor autonomía de los hijos y así en la reducción de la actividad sexual ${ }^{15,16}$.

La mejor comunicación entre padres e hijos se asocia a menor actividad sexual de los hijos y mayor uso de anticonceptivos ${ }^{17-19}$. Sin embargo, otros estudios muestran que la familia no juega un rol socializador importante y que los pares y los medios de comunicación son mucho más relevantes en el intercambio de la información sexual ${ }^{19,20}$.

Las adolescentes cuyas madres estaban solteras al nacimiento de su primer hijo están en mayor riesgo de iniciar actividad sexual a edades más tempranas, porque las hijas ven a sus madres más permisivas a la actividad sexual prematrimonial, independiente de las reales creencias sostenidas por las madres ${ }^{21-24}$.

Los hijos que viven con ambos padres experimentan menos actividad sexual e inician actividad sexual a edades mayores ${ }^{24-26}$. Mientras que el divorcio o separación de los padres puede influir en el inicio temprano cuando los padres, especialmente la madre, establece una nueva relación de pareja que incluye la actividad sexual ${ }^{26-28}$.

Los adolescentes que tienen padres más educados tienen menos riesgo de inicio sexual temprano y esto porque los padres con mayor educación tienen mejores aspiraciones educacionales para sus hijos y reconocen la dificultad de combinar logros educacionales y maternidad temprana, desanimando la actividad sexual en sus hijas ${ }^{29}$.

Los adolescentes que perciben a sus padres o muy estrictos o muy permisivos tienen más probabilidad de iniciar actividad sexual, que aquellos que perciben a sus padres como moderadamente estrictos $^{30,31}$. El empleo maternal fuera del hogar aumenta el riesgo de actividad sexual temprana en las hijas porque las madres al no estar presentes ejercen menos control, dando a las adolescentes mayores oportunidades de encuentros sexuales en sus propias casas ${ }^{29,30}$.

Finalmente, la evidencia demuestra que cuando el nivel socio-económico decrece, las tasas de actividad sexual y embarazo adolescente aumentan debido a que la pobreza, las condiciones económicas inseguras y las bajas expectativas desaniman a las adolescentes de familias pobres a transitar hacia la adultez a través de los logros educacionales y de carrera y, por lo tanto, postergar la maternidad adolescente $^{31,32}$.

En Chile no hay estudios publicados de factores familiares que se asocian al inicio sexual temprano en adolescentes y los resultados de estudios realizados en países desarrollados pueden no ser aplicables a la realidad sociocultural chilena.

El objetivo de este estudio fue determinar la asociación entre los factores familiares y el inicio de la actividad sexual temprana, en adolescentes consultante en un centro de salud sexual y reproductiva universitario con una perspectiva de género.

\section{Material y Método}

Diseño de estudio transversal analítico, que analizó los diversos factores familiares previamente identificados en otros estudios en la literatura, asociados a la actividad sexual temprana (antes de los 15 años) y a la postergación en adolescentes. La población del estudio fueron adolescentes de ambos sexos entre 12 y 19 años de la Región Metropolitana que han consultado en CEMERA. CEMERA es el primer y más importante centro de 
atención integral en salud sexual y reproductiva para adolescentes en el país, situado en la capital de Chile, que tiene como principal objetivo prevenir el primer embarazo. El estudio obtuvo la información de la entrevista estructurada, diseñada para propósitos clínicos y de investigación que aplicó la investigadora principal al ingreso de cada adolescente. A todos los adolescentes se les explicó la modalidad de atención y se les solicitó permiso explícito para utilizar los antecedentes que quedaron registrados en la ficha clínica para fines de investigación, asegurando el resguardo de la confidencialidad y anonimato de los datos. Lo anterior fue formalizado mediante firma del consentimiento informado especialmente diseñado para estos efectos.

Durante el período 2000 a 2007, 4.267 adolescentes consultaron en CEMERA. Fueron incluidos en este estudio adolescentes que reportaron haber tenido actividad sexual coital vaginal. Fueron excluidos del estudio, adolescentes que reportaron inicio de la actividad sexual por abuso sexual y aquellos con orientación sexual no heterosexual. Cumplidos los criterios de elegibilidad la población final fue de 3.210.

Dada la naturaleza confidencial de los servicios clínicos ofrecidos no se requirió el consentimiento parental. El estudio fue aprobado por el Comité de Ética en Seres Humanos de la Facultad de Medicina de la Universidad de Chile.

Las variables familiares analizadas fueron: filiación (vínculo legal entre padres-hijos, matrimonial y no matrimonial), tamaño de la familia (número de hijos), orden de nacimiento de los hijos, vive actualmente con ambos padres (sí, no), figura de crianza (crianza por ambos padres o no), calidad de la relación con los padres (definida por autoreporte como buena, regular, mala), comunicación familiar general (definida por autoreporte como buena, regular, mala), responsable de la supervisión (ambos padres, sólo padre, sólo madre, otro), tipo de permiso durante la semana (muy restringido, restringido, sin restricción), tipo de permiso los fines de semana (muy restringido, restringido, sin restricción), responsable de la supervisión, existencia de sanción al quebrantamiento de las normas familiares, edad paterna y materna al nacimiento del hijo, escolaridad paterna y materna (años de escolaridad cursados), trabajo de la madre fuera del hogar, antecedente de paternidad y maternidad adolescente de los padres, funcionamiento familiar (medido por test APGAR familiar; puntajes 0 a $6=$ disfuncional, 7 a 10 , normo funcional $)^{33}$. Se compararon dos grupos, los adolescentes que habían iniciado actividad sexual temprana, (hasta 14 años 11 meses 29 días) con aquellos que habían iniciado actividad sexual después a los 15 años y más. Dado que la literatura sugiere que hay diferencias en los comportamientos sexuales de hombres y mujeres, las variables fueron analizadas y estratificadas por sexo.

\section{Análisis estadístico}

Los resultados para las variables (discretas y continuas) medidas en escala numérica, fueron expresados como valores promedios y porcentajes para las medidas en escala nominal. En una primera etapa se determinó los adolescentes que habían iniciado actividad sexual antes de los 15 años y a los 15 años y más. Para el análisis bivariado se aplicaron las pruebas estadísticas Test $\chi^{2}$ y Test de Diferencia de Medias y Test de Wilcoxon para dos muestras independientes.

Posteriormente, se estratificó la muestra por sexo ajustando un modelo de regresión logística múltiple para adolescentes varones y otro para adolescentes mujeres que permitió identificar aquellas variables familiares asociadas al inicio de la actividad sexual temprana diferenciada por sexo. Para este análisis se incluyeron en los dos modelos variables que en el análisis bivariado tuvieron un $p$-value $\leq 0,20$, de acuerdo a Hosmer y Lemeshow ${ }^{35}$. Para evaluar la bondad del ajuste de los modelos se utilizó la prueba de Hosmer y Lemeshow $^{35}$. Se utilizó software STATA 10.0.

\section{Resultados}

Los resultados corresponden a 3.210 adolescentes de ambos sexos entre 12 y 19 años. El $90,2 \%$ eran mujeres, $96 \%$ eran estudiantes. La edad promedio a la entrevista fue de 16,3 años con un promedio de 10,6 años de escolaridad. El promedio de edad de inicio de la actividad sexual fue de 15,4 años. El 24,1\% había iniciado actividad sexual antes de los 15 años y 75,9\% a los 15 años y más. El nivel socioeconómico, (usando la escala modificada de Graffar adaptada a Chile) ${ }^{36}$, se distribuyó de la siguiente manera, 3,8\% nivel alto; $61,2 \%$ medio; $33,7 \%$ bajo; $1,3 \%$ muy bajo.

El análisis bivariado mostró que las variables 
familiares categóricas asociadas al inicio sexual antes de los 15 años fueron: filiación no matrimonial, no haberse criado con ambos padres, mala relación con los padres, no vivir actualmente con ambos padres, mala relación con los padres, mala comunicación familiar, madre sola u otro responsable, supervisión sin sanción al quebrantamiento de normas, padre con antecedente de paternidad adolescente, madre con antecedente de maternidad adolescente, madre que trabaja, tamaño de la familia (número de hijos 1 ó 2) y disfunción familiar. El tipo de permiso durante la semana y para los fines de semana, orden de nacimiento y actividad del padre no fue asociado al inicio sexual temprano (Tabla 1).

La edad paterna y materna al nacimiento del hijo y la escolaridad paterna y materna no fue asociada al inicio sexual temprano (Tabla 2).

Considerando las variables que resultaron con un $p$-value $\leq 0,20$ en el análisis bivariado ${ }^{35}$ se ajustaron dos modelos de regresión logística multiple, uno para adolescentes varones y otro para adolescentes mujeres, obteniéndose los siguientes resultados:

En el modelo de regresión logística múltiple para las mujeres, resultaron significativa las siguientes variables: filiación no matrimonial que aumenta el riesgo $60 \%$ y antecedente de paternidad adolescente en el padre, que aumentó el riesgo $39 \%$, controlada por las restantes variables. La calidad del ajuste resultó con un $\mathrm{p}=0,36$ (Prueba de Hosmer y Lemeshow ${ }^{35}$ ).

En el modelo de regresión logística múltiple para los hombres, resultaron significativas las siguientes variables: Sanción al quebrantamiento de normas familiares que aumento el riesgo en 1,50 veces y permisos sin restricción en la semana que aumenta el riesgo en 1,68 veces, controladas por las restantes variables. La calidad del ajuste resultó con $\mathrm{p}=0,75$ (Prueba de Hosmer y Lemeshow ${ }^{35}$ ) (Tabla 3).

\section{Discusión}

Concordante con lo reportado en estudios en países desarrollados, hay variables familiares que mostraron asociación con el inicio sexual temprano en esta población adolescentes, mostrando diferencias por género. Sanción al quebrantamiento de las normas familiares y tipo de permiso sin restricción durante la semana resultó significativa sólo para los hombres. Mientras que filiación no matrimonial de los padres y antecedente de paternidad adolescente en el padre, se asoció al inicio sexual temprano sólo para las mujeres.

Contrario a lo reportado en la literatura, la escolaridad del padre y de la madre, no se asoció a la postergación del inicio sexual ni en hombres ni en mujeres.

Concordante con otros estudios ${ }^{24,26}$, la no crianza por ambos padres no se asoció al inicio sexual temprano. Los permisos sin restricción durante el fin de semana no aparece como factor de riesgo, no concordante con lo reportado en la literatura que reporta que los adolescentes que perciben a sus padres o muy estrictos o muy permisivos tienen más riesgo de inicio de actividad sexual temprana ${ }^{30,31}$.

El empleo materno fuera del hogar se asoció al inicio sexual temprano en hombres y en mujeres concordante con lo ya descrito por los estudios en que señalan que el empleo materno fuera del hogar expone a mayor riesgo de actividad sexual temprana especialmente en las hijas ${ }^{29,30}$. Por otra parte, la mala comunicación familiar, mala relación padres-adolescente, disfunción familiar, resultaron asociados al inicio sexual temprano en nuestro estudio, concordante con lo reportado por la literatura. Contrario a lo encontrado por algunos autores ${ }^{11,15}$, el mayor número de hijos en la familia y la historia de maternidad adolescente en la madre no expusieron a adolescentes de ambos sexos al inicio sexual temprano.

Este estudio tiene limitaciones: considerando que el inicio sexual temprano es un fenómeno multifactorial y los factores personales, entre otros, juegan también un rol importante, el presente análisis sólo se centró en los factores familiares. Sin embargo, futuras investigaciones incluirán y profundizaran en estos otros factores. Por otro lado, los resultados son aplicables a adolescentes de la Región Metropolitana de nivel socioeconómico de nivel medio y bajo que solicitaron atención en salud sexual y reproductiva en un centro especializado, por lo que no pueden ser generalizados a la población adolescente de otros estratos socioeconómicos ni de otras regiones. Sin embargo, a pesar de estas limitaciones, nuestros hallazgos son relevantes ya que la población adolescente estudiada corresponde a una población vulnerable y en necesidad de intervenciones destinadas a prevenir 
Factores familiares asociados al inicio sexual temprano en adolescentes - E. González et al

Tabla 1. Distribución porcentual de las variables familiares categóricas asociadas al inicio temprano de la actividad sexual en adolescentes $(n=3.210)$

\begin{tabular}{|c|c|c|c|c|}
\hline Variables & Categorías & $<15$ años & $\geq 15$ años & p-value \\
\hline \multirow[t]{2}{*}{ Filiación del hijo } & Matrimonial & 70,4 & 79,2 & 0,000 \\
\hline & No matrimonial & 29,6 & 20,8 & \\
\hline \multirow[t]{2}{*}{ Figura de crianza } & Ambos padres & 63,9 & 69,6 & 0,003 \\
\hline & No ambos padres & 36,1 & 30,4 & \\
\hline \multirow[t]{3}{*}{ Relación con los padres } & Buena & 45,1 & 49,3 & \\
\hline & Regular & 40,8 & 40,8 & 0,003 \\
\hline & Mala & 14,1 & 9,9 & \\
\hline \multirow[t]{2}{*}{ Vive actualmente con } & Ambos padres & 46,2 & 53,5 & 0,001 \\
\hline & No ambos padres & 53,8 & 46,5 & \\
\hline \multirow[t]{3}{*}{ Comunicación familiar } & Buena & 49,0 & 50,6 & \\
\hline & Regular & 27,7 & 31,7 & 0,002 \\
\hline & Mala & 23,3 & 17,7 & \\
\hline \multirow[t]{4}{*}{ Responsable de supervisión } & Ambos padres & 29,9 & 35,7 & \\
\hline & Padre & 10,1 & 11,5 & 0,002 \\
\hline & Madre & 47,3 & 43,5 & \\
\hline & Otro & 12,7 & 9,4 & \\
\hline \multirow{2}{*}{$\begin{array}{l}\text { Sanción al quebrantamiento de las normas } \\
\text { familiares }\end{array}$} & Sí & 28,8 & 33,8 & 0,012 \\
\hline & No & 71,2 & 66,2 & \\
\hline \multirow[t]{3}{*}{ Tipo de permisos semana } & Muy restringido & 48,3 & 50,4 & \\
\hline & Restringido & 5,2 & 3,7 & 0,151 \\
\hline & Sin restricción & 46,5 & 45,9 & \\
\hline \multirow[t]{3}{*}{ Tipo de permisos fin semana } & Muy restringido & 9,5 & 8,8 & \\
\hline & Restringido & 56,8 & 59,5 & 0,441 \\
\hline & Sin restricción & 33,7 & 31,7 & \\
\hline \multirow[t]{2}{*}{ Paternidad adolescente del padre } & Sí & 19,7 & 14,8 & 0,002 \\
\hline & No & 80,3 & 85,2 & \\
\hline \multirow[t]{2}{*}{ Maternidad adolescente de la madre } & Sí & 40,8 & 34,5 & 0,002 \\
\hline & No & 59,2 & 65,5 & \\
\hline \multirow[t]{2}{*}{ Trabajo materno fuera del hogar } & Sí & 59,6 & 54,0 & 0,009 \\
\hline & No & 40,4 & 46,0 & \\
\hline \multirow{4}{*}{$\begin{array}{l}\text { Tamaño de la familia } \\
\text { (número de hijos) }\end{array}$} & 1 & 9,3 & 7,2 & \\
\hline & 2 & 30,7 & 28,0 & 0,015 \\
\hline & 3 & 28,2 & 33,7 & \\
\hline & 4 y más & 31,8 & 31,1 & \\
\hline \multirow[t]{4}{*}{ Orden de nacimiento } & $1^{\circ}$ & 42,5 & 42,7 & \\
\hline & $2^{\circ}$ & 28,9 & 27,9 & 0,367 \\
\hline & $3^{\circ}$ & 15,1 & 17,4 & \\
\hline & $4^{\circ}$ y más & 13,5 & 12,0 & \\
\hline \multirow[t]{2}{*}{ Funcionamiento familiar } & Disfuncional & 46,4 & 39,9 & 0,019 \\
\hline & Funcional & 53,6 & 60,1 & \\
\hline \multirow[t]{3}{*}{ Actividad del padre } & Obrero, no calificado & 45,8 & 47,5 & 0,639 \\
\hline & Empleado, independiente & 49,5 & 47,4 & \\
\hline & Profesional & 4,7 & 5,2 & \\
\hline
\end{tabular}


Factores familiares asociados al inicio sexual temprano en adolescentes - E. González et al

Tabla 2. Distribución de las variables familiares continuas asociadas al inicio temprano de la actividad sexual (promedios)

\begin{tabular}{|lccccc|}
\hline Variables & \multicolumn{2}{c}{$<\mathbf{1 5}$ años } & \multicolumn{2}{c|}{$\mathbf{1 5}$ años } & p-value \\
& Promedio & DS & Promedio & DS & \\
\hline Edad paterna al nacimiento del hijo & 27,9 & 6,97 & 28,1 & 6,86 & 0,32 \\
\hline Edad materna al nacimiento & 25,2 & 6,24 & 25,5 & 6,04 & 0,10 \\
\hline Escolaridad padre & 10,6 & 3,09 & 10,7 & 3,16 & 0,38 \\
\hline Escolaridad madre & 10,2 & 3,02 & 10,3 & 3,02 & 0,53 \\
\hline
\end{tabular}

Tabla 3. Ajuste de modelo Regresión Logística para variables familiares, estratificado según Sexo. Variables que resultaron significativas $(p<0,05)$

\begin{tabular}{|lccc|}
\hline Variables & p & OR & Intervalo de Confianza 95\% \\
\hline Sexo: Femenino & & & $(1,26-2,03)$ \\
Filiación (no matrimonial) & 0,000 & 1,60 & $(1,07-1,82)$ \\
Antecedente de paternidad adolescente en el padre & 0,015 & 1,39 & $(1,20-5,20)$ \\
Sexo: Masculino & & & $(1,08-6,66)$ \\
Sanción al quebrantamiento de las normas familiares & 0,014 & 2,50 & 2,68 \\
\hline Tipo de permisos semana (sin restricción) & 0,034 & & \\
\hline
\end{tabular}

el inicio sexual temprano y aunque sabemos que la conducta sexual es un fenómeno multifactorial, los factores familiares deben ser considerados tanto en las estrategias de prevención a nivel de políticas públicas como a nivel de intervención directa con adolescentes.

Es necesario también recalcar que debido el rol primordial que juegan los factores familiares adversos, además, de las necesidades afectivas no satisfechas de los adolescentes, entre otras, aparece como prioritario incluir a los padres en cualquier programa de prevención del embarazo adolescente. El objetivo principal de incluir a la familia seria fortalecerla como espacio de contención y apoyo de los hijos en una etapa de mayor vulnerabilidad como es la adolescencia.

\section{Referencias}

1. INJUV, Gobierno de Chile, VI Encuesta 2009. http:// www.injuv.gob.cl/injuv2010/encuestas_juventud [Consultado el 30 de marzo de 2012].

2. González E, Molina T, Montero A, Martínez V, Leyton C. Comportamientos sexuales y diferencias de género en adolescentes usuarios de un sistema público de salud universitario. Rev Med Chile 2007; 135: 1261-9.

3. FLACSO-Chile. Diagnóstico de la situación del embarazo en la adolescencia en Chile, 2008, Ministerio de Salud. Doc 536, UNFPA CHI 1R11A, FLACSO-Chile, 2008.

4. Molina R, Molina T, González E. Madres niñas-adolescentes de 14 años y menos: Un grave problema de salud pública no resuelto en Chile. Rev Med Chile 2007; 135: 79-86.

5. González E, Molina T, Montero A, Martínez V, Leyton C. Factores asociados a la continuidad del uso anticonceptivo en adolescentes solteras nulíparas. Rev Med Chile 2009; 137: 1187-92.

6. O"Donnell L, Donnell RC, Stueve A, Early Sexual Initiation and Subsequent Sex-Related Risks among Urban Minority Youth: The Reach for Health Study. Fam Plann Perspect 2001; 33 (6): 268-75.

7. Hofferth S, Factors affecting initiation of sexual intercourse in: Risking the future: Adolescent sexuality, pregnancy and childbearing, Washington, DC: National Academy Press, 1987; 1: 7-35.

8. SERNAM, Análisis de las conductas de las/os adolescentes Región Metropolitana, Santiago, Chile, 1997; Nº 53.

9. Molina R, Sandoval J, González E, (Eds.) Salud Sexual y 
Reproductiva en la Adolescencia. Santiago, Chile: Editorial Mediterráneo; 2003, p. 415-560, caps 29-40.

10. Forste R, Heator $\mathrm{T}$ B. Initiation of sexual activity among female adolescents. Youth and Society 1988; 19: 250-68.

11. Thornton A, Camburn D. The influence of the family on premarital sexual attitudes an behavior. Demography 1987; 24 (3): 323-40.

12. Miller CB, Moore K. Adolescent sexual behavior, pregnancy and parenting: Research through the 1980s. J Marriage Fam 1990; 1025-44.

13. McBride M V. Black adolescent females: A comparison of early versus late coital initiators. Fam Relat 1994; 43 (3): 342-8.

14. Lammers C, Ireland M, Resnick M, Blum R. Influences on adolescents' decision to postpone onset of sexual intercourse: A survival analysis of virginity among youths aged 13 to 18 years. J Adolesc. Health 2000; 26 (1): 42-8.

15. Hoogan PD, Kitawua ME. The impact of social status, family structure, and neighborhood on the fertility of Black adolescents. Am J Soc 1985; 90 (4): 825-55.

16. Mueller KE, Powers WG. Parent-child sexual discussion: Perceived communicator style and subsequent behavior. Adolescence 2000; 25: 469-82.

17. Fox Greer L, Inazu J.K. Patterns and outcomes of mother-daughter communication about sexuality. J Soc Issues 1980; 36: 7-29.

18. Montenegro AH. Educación sexual de niños y adolescentes. Rev Med Chile 2000; 128 (6): 571-3.

19. Fernández L, Bustos L, González L, Palma D, Villagrán J, Muñoz S. Creencias, actitudes y conocimientos en educación sexual. Rev Med Chile 2000; 128 (6): 574-83.

20. Miller CB, Moore K. Adolescent sexual behavior, pregnancy and parenting: Research through the 1980s. J Marriage Fam 1990; 1025-44.

21. Miller SK, Forehand R, Kotchick B. Adolescent sexual behavior in two ethnic minors samples: The role of family variables, J Marriage Fam 1999; 61 (1): 85-98.

22. Miller B, Sneesby KR. Educational correlates of adolescent's sexual attitudes and behavior. J Youth Adolesc 1988; 17: 521-30.

23. Kahn J, Smith K, Roberts E. Family communication and adolescent sexual behavior. American Institute for Research, Cambridge. MA, 1984.
24. Ellis BJ, Bates JE, Dodge KA, Fergusson DM, Horwood LF. Does father absence place daughters at special risk for early sexual activity and teenage pregnancy?. Child Development 2003; 74 (3), 801-21.

25. O'Connell M, Rogers C. Out-of-wedlock births, premarital pregnancies and their effect on family formation and dissolution. Fam Plann Perspect1984; 16: 157-62.

26. Newcomer S, Udry JR. Mothers' influence on the sexual behavior of their teenage children, J Marriage Fam, 1984, 46: 477-85.

27. Newcomer S, Udry JR. Parental marital effects on adolescent sexual behavior. J Marriage Fam 1987; 49: 235-40.

28. Forste R, Heaton TB. Initiation of sexual activity among female adolescents. Youth and Society 1988; 19: 250-68.

29. Davis EC, Friel LV. Adolescent sexuality: Disentangling the effects of family structure and family context. J Marriage Fam 2001; 63 (3): 669-81.

30. Gerard JM, Krishnakumar A, Buehler C. Marital conflict, parent-child relations, and youth maladjustment. J Family Issues 2006; 27 (7): 951-75.

31. Kaye K, Moore KA, Hair EC, Hadley A, Day R. Orthner D. Parent marital quality and the parent-adolescent relationship: Effects on sexual activity among adolescents and youth. Marriage and Family Review 2009; 45 (2-3): 270-88.

32. Hutchinson MK, Jemmott JB, Jemmott LS, Braverman P, Fong GT. The role of mother-daughter sexual risk communication in reducing sexual risk behaviors among urban adolescent females: A prospective study. J Adolesc Health 2003; 33: 98-107.

33. Smilkstein G. The Family APGAR: A proposal for family function test and its use by physicians. J Fam Pract 1978; 6 (6): 1231-9.

34. INE. Proyección de población por grupo edad, según región, servicio de salud, comuna y sexo. http://www.ine. cl/canales/chile_estadístico/censo_población_vivienda [Consultado el 10 de marzo de 2012].

35. Hosmer DW, Lemeshow S. Applied Logistic Regression, $2^{\text {nd }}$ Edition, Ed. New York: John Wiley \& Sons. INC. 2000.

36. Álvarez M, Muzzo S, Ivanovic D. Escala para medición del nivel socioeconómico en el área de salud. Rev Med Chile 1985; 113: 243-9. 